\title{
Estudo comparativo da reabsorção radicular apical em pacientes bruxônomos e pacientes sem sinais clínicos de desgaste dentário*
}

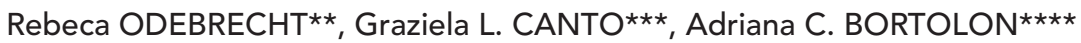

\begin{abstract}
Resumo
A reabsorção radicular, assim como o bruxismo, são temas amplamente abordados na literatura, contudo, a associação dos mesmos ainda permanece pouco documentada. Desse modo, esta pesquisa objetiva comparar pacientes portadores de bruxismo e pacientes sem sinais clínicos de desgaste dentário em relação à quantidade de reabsorção radicular apical nos incisivos superiores. Para tanto foram avaliados clínica e radiograficamente 64 pacientes, perfazendo um total de 256 incisivos acompanhados. Os resultados demonstram que há uma correlação positiva entre bruxismo e reabsorção radicular.
\end{abstract}

Palavras-chave: Bruxismo. Reabsorção radicular.

\section{INTRODUÇÃO}

O bruxismo é um termo usado para indicar a ação de cerrar ou ranger os dentes, em atividades não funcionais do sistema mastigatório, tendo sido descrito pela primeira vez por Marie e Pietwiekwicz ${ }^{20}$. Estes caracterizaram-no como um hábito parafuncional, diurno ou noturno, geralmente inconsciente, produzindo sons audíveis, os quais são impossíveis de serem reproduzidos pelo paciente conscientemente.

Contudo, o bruxismo não é um problema recente da civilização. Este fenômeno patológico foi citado em Salmos de Davi e Mateus (A Bíblia Sagrada - Mateus, cap. 24;51), que diziam: "E removê-lo-á e porá a sua parte com os hipócritas e haverá choro e ranger dos dentes".
Em 1966, Nadler ${ }^{21}$ descreveu o bruxismo como uma disfunção que afeta uma grande porcentagem da população, e em todas as faixas etárias; além disso, relatou que a etiologia pode ser de natureza local, sistêmica, de ordem psicológica ou ocupacional. Nadler ${ }^{21}$ salientou que o fator psicológico parece ser a causa principal e considerou, ainda, que fazem parte da categoria do bruxismo todos os hábitos de apertamento e rangimento de dentes.

Em relação à incidência do bruxismo na população, Ahmad² afirma ser possível encontrar sinais da disfunção em $81 \%$ em crianças. Já Schneider e Peterson ${ }^{24}$ relataram encontrar o bruxismo variando entre 8 e $15 \%$ na população, observando, ainda, que ocorre um aumento na incidência do

* Trabalho desenvolvido para o Programa de Apoio à Pesquisa - CNPq

** Especialista em Odontopediatria pela UFSC e Estagiária da disciplina de Oclusão da UFSC

*** Professora Assistente do Curso de Graduação em Odontologia da Universidade Federal de Santa Catarina/Mestre em Ortodontia pela FOB/USP.

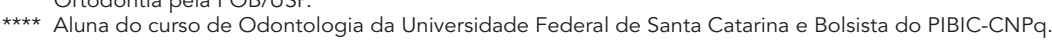


bruxismo na infância até a adolescência e a partir daí uma diminuição relacionada com o aumento da maturidade e uma adaptação do estresse psicoemocional do paciente.

A exemplo do bruxismo, a reabsorção radicular dos dentes permanentes constitui uma alteração amplamente discutida na literatura, contudo, a sua etiologia ainda é uma questão controversa dentro da Odontologia.

Dentre os fatores relacionados ao surgimento e progressão das reabsorções radiculares destacam-se os processos inflamatórios periapicais, as compressões císticas e as de dentes impactados ${ }^{22}$, as deficiências metabólicas ${ }^{10}$, os distúrbios hormonais ${ }^{19} \mathrm{e}$ as forças impostas pela movimentação ortodônti$\mathrm{ca}^{9}$. A susceptibilidade individual é considerada o fator principal no potencial à reabsorção radicular. É um enigma que em alguns pacientes os dentes podem ser movidos $20 \mathrm{~mm}$ sem evidenciar radiograficamente reabsorção radicular, enquanto em outros pacientes uma mera extrusão de 2 a $3 \mathrm{~mm}$ pode resultar em extensa reabsorção radicular apical $^{1,3,5,11,13}$. Dessa forma, o processo de reabsorção radicular varia entre as pessoas e na mesma pessoa em momentos diferentes.

Apesar da maioria dos autores acreditar que as forças aplicadas aos dentes, por meio dos aparelhos ortodônticos, são as grandes responsáveis pelo desencadeamento do processo de reabsorção, Harris e Butler ${ }^{12}$ afirmaram que o tratamento ortodôntico, por si só, não é o maior contribuinte para a reabsorção. Existem outros fatores, os quais associados à aplicação de força, desencadeiam o processo. Baseados na constatação de que geralmente a força é suave e na premissa de que reabsorção radicular provocada pelo tratamento ortodôntico cessa no momento em que a mesma é removida, há concordância de que as melhoras estéticas e funcionais justificam os riscos.

$\mathrm{Na}$ tentativa de descobrir fatores que, coadjuvantes ao tratamento ortodôntico, poderiam induzir o processo de reabsorção alguns trabalhos foram realizados, como o de Harris e Butler ${ }^{12}$, que obser- varam que inúmeras pessoas portadoras de bruxismo tendiam a mostrar dentes mais curtos, devido à compressão do ligamento periodontal resultante de uma carga oclusal excessiva, sugerindo a existência da relação entre bruxismo e reabsorção radicular.

Portanto, esse estudo foi realizado com o objetivo de elucidar a dúvida de Harris e Butler ${ }^{12}$, comparando pacientes portadores de bruxismo e pacientes sem sinais clínicos de desgaste dentário, em relação à quantidade de reabsorção radicular apical nos incisivos superiores.

\section{MATERIAL E MÉTODOS Material}

Para verificar a quantidade de reabsorção radicular em pacientes com bruxismo, foi compulsada a documentação clínica de 64 pacientes, com idade entre 18 e 41 anos, sem distinção de raça ou gênero e independentemente do tipo de má oclusão apresentada, divididos em dois grupos: o grupo 1 , formado por 32 pacientes pré-examinados, bruxônomos, e integrantes da Clínica ou alunos do Curso de Graduação em Odontologia da Universidade Federal de Santa Catarina. A procedência dos pacientes do grupo 2 foi a mesma do grupo 1, porém estes não apresentavam sinais clínicos de desgaste dentário. Todos os pacientes, de ambos os grupos, foram selecionados e pré-examinados por um professor da disciplina de Oclusão.

Para a seleção da amostra, os pacientes cujos dentes apresentavam tratamento endodôntico diagnosticado nas radiografias ou presença de prótese fixa foram excluídos. Também foram eliminadas as radiografias que evidenciavam distorção de imagem ou falta de nitidez. Pacientes em período de gestação, foram excluídas da pesquisa para evitar exposição à radiação, mesmo que essa seja comprovadamente pequena. Em função da alta relação reabsorção radicular/tratamento ortodôntico, não foram utilizados nessa pesquisa pacientes que realizaram tratamento ortodôntico prévio.

Para a quantificação de reabsorção radicular, foram examinadas apenas as radiografias dos incisivos 
superiores. Duzentos e cinqüenta e seis dentes foram analisados. Optou-se por trabalhar somente com esse grupo de dentes, por que a maioria dos autores concordam que são esses os dentes atingidos com mais freqüência e intensidade por reabsorção radicular apical, com causas patológicas ${ }^{1,3,7,15,16}$.

\section{MÉTODOS \\ Radiografias}

As radiografias periapicais foram obtidas por um único operador pela técnica do paralelismo (cone longo). As angulações aferidas ao cabeçote do aparelho de raio $\mathrm{X}$ foram determinados pelo uso de um posicionador intra-bucal. O tempo de exposição à radiação foi de 0,8 segundos.

Todos os pacientes que participaram dessa pesquisa foram adequadamente protegidos da radiação secundária por colete de chumbo e todas as radiografias periapicais foram reveladas manualmente na câmara escura, pelo mesmo operador. Além disso foi elaborado um documento com os termos de Consentimento Livre e Esclarecido, que foi assinado pelos integrantes da pesquisa (pacientes), de acordo com as normas da Comissão de Ética.

Para a quantificação da reabsorção radicular apical, a exemplo de outros pesquisadores, foi utilizado o método proposto por Levander, Malmgreen e
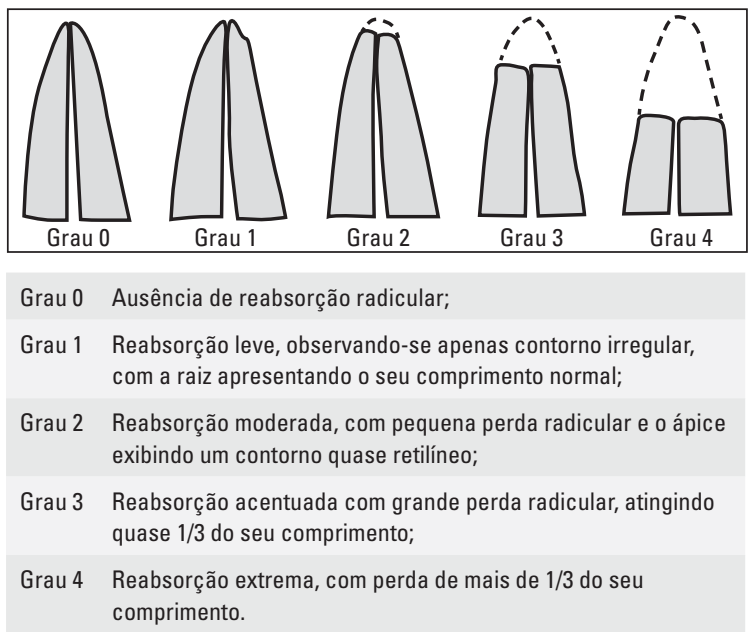
com a raiz apresentando o seu comprimento normal;

Grau 2 Reabsorção moderada, com pequena perda radicular e 0 ápice exibindo um contorno quase retilíneo;

Grau 3 Reabsorção acentuada com grande perda radicular, atingindo quase $1 / 3$ do seu comprimento;

Grau 4 Reabsorção extrema, com perda de mais de 1/3 do seu comprimento.

FIGURA 1 - Classificação dos graus de reabsorção segundo Levander, Malmgreen e Eliasson ${ }^{18}$.
Eliasson ${ }^{18}$ que classificaram a reabsorção radicular em 5 graus (Fig. 1).

A análise radiográfica foi realizada em sala escurecida, na qual a única fonte de luz provinha do negatoscópio. As radiografias foram colocadas em cartões, dispostas sobre o negatoscópio e cobertas com anteparo de cartolina preta, com uma abertura central menor que o tamanho de uma película radiográfica. Desse modo, pôde-se visualizar uma de cada vez, sem influência da luz externa e sem que o examinador observasse a coroa dos dentes (uma vez que o desgaste na coroa, quando presente, poderia influenciar no julgamento). Os cartões apresentavam somente um código de identificação, desconhecido pelo examinador, ou seja este não sabia qual grupo o paciente integrava, sendo por isso um estudo cego. A análise foi realizada, por um único examinador, professor do curso de Odontologia da Universidade Federal de Santa Catarina. A classificação do grau de reabsorção de cada dente foi anotada individualmente na ficha de cada paciente.

\section{Erro do Método (Erro Intra-Examinador)}

Dezesseis casos foram selecionados aleatoriamente (oito de cada grupo) e medidos novamente pelo mesmo examinador. As medidas repetidas foram testadas pelo coeficiente de Kappa Ponderado. O coeficiente de Kappa Ponderado, demonstrou ser um excelente nível de calibração intra-examinador (Tab. 1).

\section{ANÁLISE ESTATÍSTICA}

$\mathrm{Na}$ análise das radiografias periapicais, a quantidade de reabsorção entre os dois grupos: 1 (controle), e 2 (bruxismo), foi comparada por meio do teste não-paramétrico de Kruskal-Wallis.

\begin{tabular}{|c|c|c|}
\hline \multicolumn{3}{|c|}{ Tabela 1 - Erro intra-examinador. } \\
\hline Grupos & Valor do coeficiente & $\mathrm{P}$ \\
\hline 1 & 0,6184 & $>6$ \\
2 & 0,8255 & $>6$ \\
Geral & $\mathbf{0 , 7 4 4 6}$ & $>6$ \\
\hline
\end{tabular}


Para a verificação da possibilidade de erro, quando da atribuição dos escores, e também a soma dos postos de ambos os grupos, foi utilizado o teste não-paramétrico de Mann-Whitney.

\section{RESULTADOS}

\section{Comparações entre os Grupos}

Após análise cuidadosa das radiografias, e classificação com os respectivos escores dos graus de reabsorção radicular, os dados foram submetidos à avaliação estatística.

No grupo 1 (controle), formado por pacientes sem sinais clínicos de desgaste dentário, 128 dentes foram analisados, dos quais apenas dois apresentaram reabsorção moderada (grau 2), ao passo que a maioria (81 dentes) não apresentou qualquer sinal de reabsorção radicular apical (Tab. 2, Fig. 2).

No grupo 2 (bruxismo), formado por pacientes com sinais clínicos de desgaste dentário, também 128 dentes compunham o grupo, e, entre estes, 44 não apresentaram reabsorção (escore 0); 66 reabsorveram levemente (escore 1) e 18 dentes tiveram reabsorção moderada (escore2), (Tab. 2, Fig. 2).

O grau de certeza, ou possibilidade de erro, as-

\begin{tabular}{|c|c|c|c|c|c|c|}
\hline \multicolumn{7}{|c|}{$\begin{array}{c}\text { Tabela } 2 \text { - Escores obtidos nos } \\
\text { grupos } 1 \text { (controle) e } 2 \text { (bruxismo) }(n=256) \text {. }\end{array}$} \\
\hline \multirow[t]{3}{*}{ GRUPOS } & \multicolumn{6}{|c|}{ ESCORES } \\
\hline & \multicolumn{2}{|c|}{0} & \multicolumn{2}{|c|}{1} & \multicolumn{2}{|c|}{2} \\
\hline & $\mathrm{n}$ & $\%$ & $\mathrm{n}$ & $\%$ & $\mathrm{n}$ & $\%$ \\
\hline 1 & 81 & 63,28 & 45 & 35,16 & 2 & 1,56 \\
\hline 2 & 44 & 34,37 & 66 & 51,56 & 18 & 14,07 \\
\hline
\end{tabular}

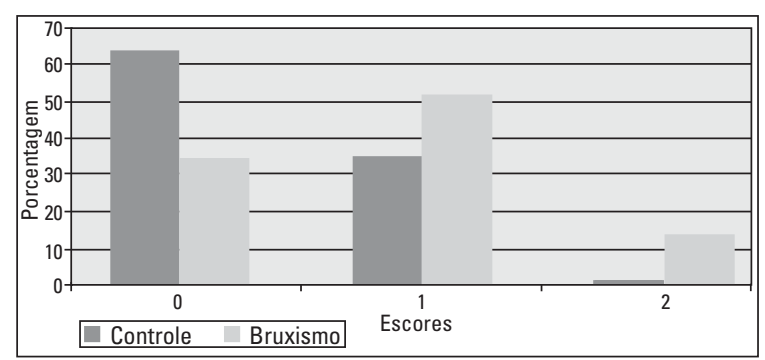

FIGURA 2 - Porcentagem de dentes classificados com escore 0, 1 e 2 nos dois grupos estudados $(\mathrm{n}=256)$.

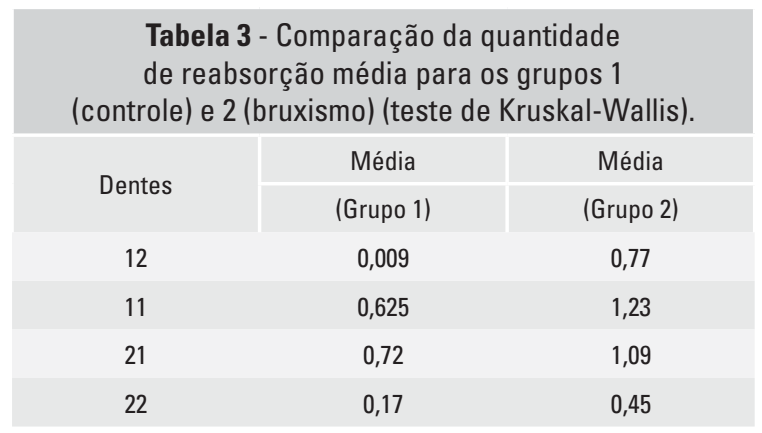

$T=78,63(P<0,001)$

sim como a respectiva soma dos postos do primeiro grupo e do grupo 2, obtidos pelo teste de MannWhitney, demonstrou que todos os grupos encontram-se num intervalo considerado bom, quando comparados ao padrão, que para a possibilidade de erro é $\mathrm{P}<0,05$. Por exemplo, para Incisivo Lateral Superior Direito (12), quando analisado o grupo 1 (controle) versus o grupo 2 (bruxismo), a possibilidade de certeza de que o segundo grupo reabsorve mais que o primeiro foi de $99,9 \%$ (Tab. 3).

\section{DISCUSSÃO}

Apesar do bruxismo ser um assunto de conhecimento e discussão não muito recente, não existe até o presente momento uma publicação que venha a descrever com clareza a sua relação com o processo de reabsorção radicular apical. Por esse motivo, serão abordadas, de maneira breve, algumas considerações de como ocorre o processo de reabsorção radicular, na tentativa de correlacionar esse comportamento das estruturas dentárias ao hábito parafuncional de ranger os dentes.

\section{Algumas considerações sobre bruxismo e reabsorção radicular}

Arnold ${ }^{4}$ relatou que pacientes com bruxismo severo podem, com o passar do tempo, apresentar desaparecimento da lâmina dura dentária, reabsorções radiculares e lesões periapicais ou periodontais.

Crothers $^{8}$ afirmou que o desgaste dentário é 
uma associação entre o estado da dentição, meio ambiente, dieta alimentar erosiva, regurgitação crônica e atrição devido ao bruxismo.

Segundo Clarke, Townsend e Carey ${ }^{6}$, em média, os bruxônomos típicos tem episódios de 58 segundos de apertamento dos dentes, gerando uma força substancial sobre estes.

Com a persistência do bruxismo, o ligamento periodontal pode ser danificado, aumentando assim a mobilidade dentária. A pressão nos dentes pode interferir no aporte sangüíneo local e levar à perda de osso alveolar ${ }^{23}$.

Outros estudos indicam que a reabsorção dos tecidos dentinários mineralizados tem início quando os osteoclastos conseguem acesso aos tecidos mineralizados por uma lesão na camada das células germinativas que recobrem o tecido quando o pré-cemento é lesado mecanicamente ${ }^{14,25}$.

A reabsorção radicular não se inicia no centro da zona hialinizada, mas na periferia, onde as células viáveis ainda existem. Duas condições são necessárias para que a reabsorção ocorra: dano na barreira do pré-cemento e presença de células viáveis, disponíveis para invadir e remover o tecido necrótico que cobre a superfície.

\section{Considerações sobre a amostra e o método}

A amostra foi constituída por pacientes selecionados pelo mesmo operador e a faixa etária foi semelhante para os dois grupos, 1 (controle) e 2 (bruxismo), eliminando possíveis influências da idade na quantidade de reabsorção encontrada. Linge B. e Linge L. ${ }^{19}$ afirmaram que a idade reduz o problema de crescimento residual, o que pode interferir na quantidade de reabsorção.

O método radiográfico adotado (radiografias periapicais) constitui o método de escolha para o estudo da reabsorção radicular apical, e por isso é utilizada pela maioria dos autores ${ }^{16,17,19}$. A técnica fornece as informações mais apropriadas: oferece menor radiação ao paciente, quando utilizada nos dentes que comumente apresentam maior encurtamento, ou seja os incisivos; provoca menos distorção e erros de superposição também são menores, quando comparadas às radiografias panorâmicas e cefalométricas. Com a utilização da técnica do paralelismo, há uma menor absorção de radiação pelos tecidos radiossensíveis da cabeça e do pescoço, em comparação à técnica periapical da bissetriz, cefalométrica e radiografia panorâmica.

O método que classifica a reabsorção por escores, proposto por Levander e Malmgreen ${ }^{17}$, embora subjetivo, é utilizado com freqüência no estudo da reabsorção radicular. Sua principal vantagem é o fato de não depender da padronização da radiografia inicial. Entretanto, todas as radiografias periapicais, foram realizadas e reveladas pelo mesmo operador.

Além disso o erro intra-examinador, neste estudo, demonstrou excelente nível de concordância, reduzindo a possibilidade de erro.

\section{Interpretação dos resultados Comparação entre os Grupos}

Quando feita a comparação entre o grupo 1 e 2 (controle X bruxismo) (Tab. 3), observou-se que a reabsorção média para o segundo foi significativamente maior do que para o primeiro, sugerindo com isso que realmente o hábito de ranger os dentes pode influenciar ou acarretar uma certa quantidade de reabsorção radicular apical como já havia sido sugerido, em 1992 por Harris e Butler ${ }^{12}$.

\section{CONCLUSÃO}

De acordo com a metodologia utilizada e com os resultados obtidos, em relação à quantidade de reabsorção radicular decorrente do estudo de pacientes portadores ou não de bruxismo, pode-se concluir que o grupo 1 (denominado de controle), que continha pacientes sem sinais clínicos de desgaste dentário, teve uma quantidade de reabsorção radicular apical significativamente menor que o grupo 2 (composto pelos pacientes portadores de bruxismo).

Enviado em: Junho de 2001

Revisado e aceito: Setembro de 2001 


\title{
Comparative study of apical root resorption in patients whith bruxism and without clinical signs of dental wearing
}

\begin{abstract}
Root resorption and bruxism are largely study in literature. However their association still remains few documented. The aim of this study is compare the quantity of apical root resorption in upper permanent incisors in patients with bruxism and without clinical signs of dental wearing. 64 patients (256 incisors) were evaluated clinical and radiografically. The results shows the correlation between bruxism and root resorption.
\end{abstract}

Key words: Bruxism. Root resorption.

\section{REFERÊNCIAS}

1. AHLGREN, J. A ten-year evaluation of the quality of orthodontic treatment. Swed Dent J, Stockholm, v. 17, no. 5, p. 201-209, 1993.

2. AHMAD, R. Bruxism in children. J Periodontol, Birmingham, v. 37, p. 311 , July/Aug. 1966.

3. ALEXANDER, S. A. Levels of root resorption associated with continuous archectional arch mechanics. Am J Dentofacial Orthop Orthod, St. Louis, v.110, no. 3, p. 321-324, Sept. 1996.

4. ARNOLD, M. Bruxism and occlusion. Dent Clin North Am, Philadelphia, v. 35, no. 3, p. 395-407, 1981.

5. ATTANASIO, R. Nocturnal bruxism and its clinical management. Dent Clin North Am, Philadelphia, v. 35, no. 1, p. 245252,1991

6. CLARKE, N. G.; TOWNSEND, G. C.; CAREY, S. E. Bruxism patterns in man during sleep. J Oral Rehabil, Oxford, no. 11, p. 198-208, 1979.

7. COPELAND, S.; GREEN, L. J. Root resorption in maxillary central incisors following active orthodontic treatment. Am J Orthod, St. Louis, v. 89, p. 51-55, Jan. 1986.

8. CROTHERS, A. J. R. Tooth wear and facial morphology. Am J Dent, Chicago, v. 20, p. 333-341, 1992.

9. DESHIELDS, R. W. A study of root resorption in treated Class II, Divsion I malocclusion. Angle Orthod, Appleton, v. 39, no. 4, p. 2331-2445, Oct. 1969.

10. ENGSTROM, C.; GRANGSTROM, G.; THILANDER, B. Effect of orthodontic force on periodontal tissue metabolism: a histological and biochemical study in normal and hipocalcemic young rats. Am J Orthod Dentofacial Orthop, St. Louis, v. 93, no. 6, p. 486-495, June 1988.

11. GAGE, J. P. Diagnosis and treatment in general practice of temporomandibular joint disorders. Aust Dent J, Sydney, v. 22, p. 382-388, 1977.

12. HARRIS, E. F.; BUTLER, M. L. Patterns of incisor root resorption before orthodontic correction in cases with anterior open bites. Am J Orthod Dentofacial Orthop, St. Louis, v. 101, p. 112 119, Feb. 1992.

13. HUMSI, A. N. K. et al. The immediate effects of a stabilization splint on the muscular simmetry in the masseter and anterior temporal muscles of patients with a craniomandibular disorder J Prosthet Dent, St. Louis, v. 62, no. 3, p. 339-342, Sept. 1989.

14. JONES, S. J.; BOYDE, A. The resorption of dentine and cementum in vivo and in vitro. In: DAVIDOVICH, Z. (Ed.). Biological mechanisms of tooth eruption and root resorption. Ohio: Columbus, 1988. p. 335-354.

15. KALEY, J.; PHILLIPS, C. Factors relates to root resorption in edgewise practice. Angle Orthod, Appleton, v. 61, no. 2, p. 125-132, 1991.

16. KETCHAM, A. H. A preliminary report of investigation of apical root resorption of permanent teeth. Int J Orthod Oral Surg Radiog, St. Louis, v. 18, no. 2, p. 97-127, Feb. 1995.

17. LEVANDER, E.; MALMGREEN, O. Evaluation of the risk of the root resorption during orthodontic. Treatment: A study on upper incisors. Eur J Orthod, London, v. 10, no. 1, p. 30-38, Feb. 1988.

18. LEVANDER, E.; MALMGREEN, O.; ELIASSON, S. Evaluation of root resorption in relation to two orthodontic treatment regimes. A clinical experimental study. Eur J Orthod, London, v. 16, no. 3, p. 223-228, June 1994.

19. LINGE, B. O.; LINGE, L. Apical root resorption in upper anterior teeth. Eur J Orthod, London, v. 5, no. 3, p. 173-183, Aug. 1983.

20. MARIE, M. M.; PIETWIEKWICZ, M. La bruxomanie. Rev Stomatol, Paris, v. 14, p. 107, 1907.

21. NADLER, S. C. The effects of bruxism. J Periodontol, Birmingham, v. 37, p. 311, July/Aug. 1966.

22. PUCHE, L. A. Reabsorción radicular y ortodoncia. Acta Odontol Venez, Caracas, v. 31, n. 1, p. 44-45, abr. 1993.

23. SAYERS, E. Experimental study on bruxism. J Dent Res, Chicago, v. 2, no.10, p. 312-315, Feb. 1986.

24. SCHNEIDER, P. E.; PETERSON, J. Oral habits: considerations in management. Pediatr Clin North Am, Philadelphia, v. 29, p. 523-546, 1982.

25. TRONSTAD, L. Root resorption: a multidisciplinary problem in dentistry. In: DAVIDOVICH, Z. (Ed.). Biological mechanisms of tooth eruption and root resorption. Ohio: Columbus, 1988. p. 293-301.

\section{Endereço para correspondência}

Rebeca Odebrecht

Rua Fernando Machado n.73 sala 604 - Centro

CEP 88010-510 - Florianópolis - SC, Brasil.

e-mail: rebecaodebrecht@ig.com.br 